\title{
DES PONTS ENTRE PÉDIATRIE ET SANTÉ PUBLIQUE:
}

\section{est-ce que les médecins scolaires du 21ème siècle sont des idéalistes anachro- niques ou des innovateurs ? Un plaidoyer pour les services de santé scolaire.}

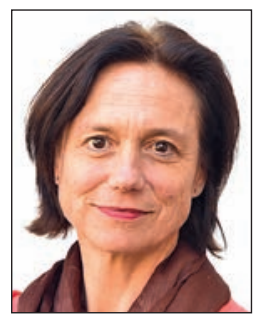

Tina Huber-Gieseke

https://doi.org/ 10.35190/f2021.4.6
Correspondance: tina.huber-gieseke @ville-fr.ch

\section{L'activité du médecin scolaire hier et aujourd'hui}

Un regard sur l'histoire des médecins scolaires (MS) montre que leur activité a toujours été étroitement liée aux conditions de vie et à la situation sanitaire des enfants, adolescent-e-s, leurs familles et leur entourage. Les MS travaillaient en réseau avec les institutions dans lesquelles se trouvent les enfants et qui agissent autour de l'enfant, et ils entretenaient le contact avec les groupes marginaux. II s'agissait en première ligne de rattraper celles et ceux qui tombaient entre les mailles du système; cela pour permettre, au moyen d'une approche à bas seuil et si nécessaire active, l'accès aux soins et à la prévention à toutes et tous, indépendamment du statut social.

Soutenir un développement sain était déjà le leitmotiv des générations précédentes de MS au siècle passé. Les défis majeurs étaient les contrôles lors de suspicion de tuberculose ou de poux, la protection contre les maladies infectieuses par les vaccinations de masse, comme aussi l'hygiène quotidienne, une alimentation suffisante et la prévention de contaminations en chaîne dues à l'habitat souvent exigu des familles. Lors du congrès sur l'hygiène en 1906 à Genève un ophtalmologue a observé: "toute école devrait avoir un médecin scolaire avec suffisamment de connaissances pour propager l'hygiène du mode de vie»1).

Les conditions de vie aujourd'hui améliorées pour la plus grande partie de la population, un système d'assurance maladie développé dans tout le pays garantissant l'accès aux soins ainsi qu'un large réseau d'aide sociale, font qu'on pourrait croire que les MS et leur personnel, au regard préventif sans être invasif, n'ont plus de travail et pourraient être remplacés par des concepts de santé.

II n'en est pas ainsi: plus qu'autrefois les MS et les services de santé scolaire se voient confrontés au défi de filtrer celles et ceux qui tombent entre les mailles du filet social, qui n'ont guère accès aux soins et à la prévention et sont donc désavantagé-e-s. On sait aussi que les enfants et adolescent-e-s sous stress chronique se développent moins bien et sont moins résilients. II suffit de penser aux enfants issus de la migration, aux enfants touchés par la pauvreté, grandissant dans une famille avec des problèmes psy- chosociaux, n'ayant pas ou ayant perdu le contact avec le/la médecin de famille. Le/la MS peut alors indiquer des pistes et contacter les collègues installé-e-s ou les psychologues scolaires, orthophonistes et assistantes sociales et assistants sociaux ${ }^{2}$.

\section{Entre scolarité et santé}

Les services de santé scolaire se trouvent à l'interface entre scolarité et santé. Les MS ont une «fonction-clé», p.ex. dans des situations complexes, exigeant des compétences en santé autant que des connaissances concernant l'école: vu le nombre croissant d'enfants avec des maladies chroniques, les MS assument un rôle important en collaborant à l'intégration de ces enfants dans l'école normale. Ils «traduisent» les besoins de l'enfant à l'intention de l'école, contribuent à l'élaboration de plans d'urgence, à mettre en place des mesures de soutien ou à clarifier des questions médicales spécifiques en vue de camps scolaires. (Figure 1)

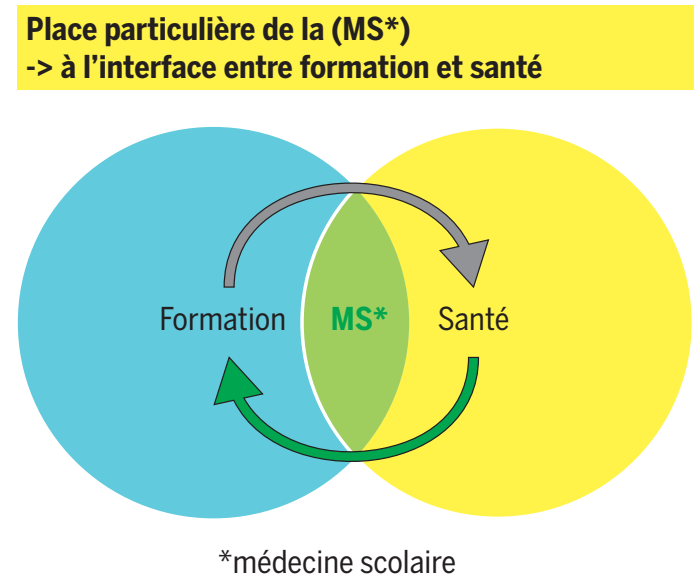

Figure 1. Place de la médecine scolaire (avec l'accord de la Dresse A. Tschumper).

\section{Collaboration et réseaux}

Les pédiatres, médecins de famille et MS sont des partenaires pour la promotion d'un développement sain des enfants et adolescent-e-s, tout en respectant le secret médical. Leurs activités sont complémentaires, elles/ils ont des positions différentes dans la chaîne de prévention et de soins. Les MS identifient 


\section{Formation continue}

des problèmes développementaux ainsi que des troubles physiques ou psychiques pas encore détectés et adressent les élèves concernés à leur médecin. En général les MS ne traitent pas elles/eux-mêmes les enfants ${ }^{3)}$. Toutes ces facettes sont typiques de l'activité de pédiatrie sociale, qui veut d'une part «filtrer» et d'autre part «rattraper». Nous souhaitons illustrer, par le modèle décrit ci-après, comment les différentes approches se conjuguent pour prendre en compte les aspects individuels de santé aussi dans le cadre scolaire. (Figure 2)

\section{Rôles, tâches et compétences des MS}

Le modèle MS en tant qu'«Expert-e en santé dans le cadre scolaire» a été adapté en se basant sur le rôle d'expert médical (CANmed $\left.{ }^{4}\right)$ de l'OMS-Euro et de l'EU$\mathrm{SUHM}^{10)}$. II présente les multiples rôles des MS et les compétences nécessaires.

Contrairement à d'autres pays, en Suisse il n'existe pas de formation ou spécialisation spécifique pour $\mathrm{MS}$, où sont transmises les compétences mentionnées dans le tableau 1. De nombreux cantons proposent leurs propres cours de médecine scolaire. Les services de santé scolaire à temps plein forment leurs nouvelles collaboratrices et nouveaux collaborateurs généralement à l'interne et organisent de plus en plus de propres formations continues. La Haute école de sciences appliquées de Zurich a développé son propre concept de curriculum pour MS. Scolarmed $\mathrm{CH}^{11)}$ organise chaque année une journée scientifique. En fait les MS suivent une formation continue régulière dans différentes disciplines, en santé publique, prévention et promotion de la santé, infectiologie, vaccinologie, médecine de l'environnement et sur des sujets pédiatriques et pédopsychiatriques, notamment pédiatrie du développement et troubles du comportement, enfin en communication. Certains services de santé scolaire plus amples couvrent la diversité interdiscipli- naire par l'engagement de médecins spécialistes des domaines santé publique, pédiatrie et pédopsychiatrie.

\section{Quels champs d'activité couvre le service}

de médecine scolaire 2021?

Exemple de cas, MS de la ville de Fribourg

Diego, 5 ans, vient d'arriver en ville avec sa mère. Sa famille est originaire du Cap Vert. Sa maman l'inscrit à l'école primaire; le point de contact de l'école pour les familles issues de la migration à Fribourg l'adresse à la MS pour l'examen d'entrée à l'école. Les parents sont séparés, le père vit à Londres. Diego a passé ses premières années de vie en Angleterre où il a fréquenté une structurepréscolaire. Sa langue maternelle est le portugais, la langue du père l'anglais. Diego ne formule que peu de phrases claires et communique avec des mots qui lui sont propres. C'est un enfant agité qui ne s'engage guère dans un échange, cherche constamment le contact avec sa mère, ne réagit paslorsqu'on lui adresse la parole. La mère signale qu'il a parlé tardivement et que les contacts sont difficiles aussi avec les autres enfants. Tout examen ou test de perception s'avère impossible. Diego fait preuve d'une nervosité frappante et d'une agitation intérieure qui se mue en peur lorsque la MS l'approche. Un comportement semblable a été observé par l'enseignante à l'école. Après plusieurs essais il est décidé de retenter l'examen trois mois plus tard.

Questions ouvertes: est-ce que Diego est agité à cause de l'absence de langage? Est-ce que l'agitation exprime de la peur? Est-ce qu'il existe un problème développemental sous-jacent? Comment procéder si la famille n'a pas encore de pédiatre? Qui coordonne la situation? Quelles sont les priorités?

L'exemple met en évidence que l'intégration des enfants, indépendamment de leur origine et environnement social, est actuellement une priorité souhaitée de la politique scolaire.

\section{1) Centré sur des aspects de santé importants au niveau scolaire}

\section{2) Réseau, collaboration et familier de l'école et de ses services, PAS d'activité curative}

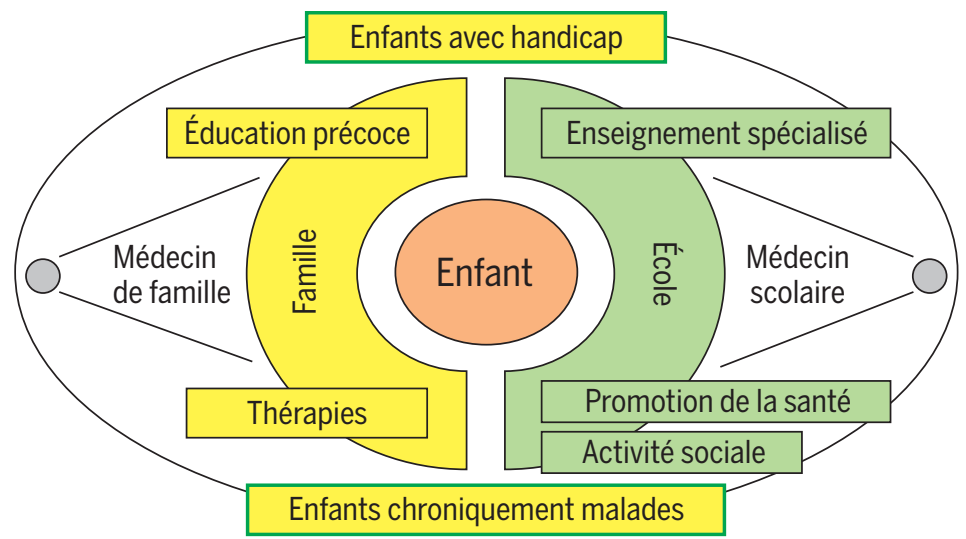

Figure 2. Collaboration médecins de famille - médecin scolaire. L'enfant est au centre (avec l'accord de la Dresse A. Tschumper). 
Formation continue

\begin{tabular}{|c|c|}
\hline Rôle & Compétences (extrait) \\
\hline Expert & $\begin{array}{l}\text { A de bonnes connaissances et compréhension: } \\
\text { - du développement biopsychosocial individuel } \\
\text { - des maladies transmissibles et non transmissibles } \\
\text { - de la prévention et des concepts de promotion de la santé individuelle } \\
\text { et collective } \\
\text { - de l'interaction entre formation et santé } \\
\text { - des influences environnmentales }\end{array}$ \\
\hline Communicateur & $\begin{array}{l}\text { - Communique respectueusement avec les enfants/adolescent-e-s/parents } \\
\text { en respectant le secret médical, ainsi qu'avec d'autres acteurs du domaine } \\
\text { de la santé, autorités et médias } \\
\text { - Mène des entretiens motivationnels }\end{array}$ \\
\hline Collaborateur & $\begin{array}{l}\text { - A la capacité de collaborer efficacement avec les collègues et au niveau } \\
\text { communal }\end{array}$ \\
\hline Gestionnaire & $\begin{array}{l}\text { - Possède une compréhension approfondie pour l'organisation des institutions } \\
\text { du domaine de la santé et de la formation } \\
\text { - A les capacités d'organiser la collaboration interdisciplinaire et de coordonner } \\
\text { les offres de soutien pour enfants et adolescent-e-s dans le cadre scolaire, } \\
\text { en gardant à l'esprit le meilleur développement possible de l'individu } \\
\text { et de la collectivité }\end{array}$ \\
\hline Promoteur de santé & $\begin{array}{l}\text { - Sur la base de ses connaissances médicales et des priorités de santé } \\
\text { de la communauté locale, des structures et agendas politiques, il s'engage } \\
\text { pour les intérêts et la promotion de la santé vis-à-vis } \\
\text { des autorités (commune, canton, école) ainsi qu'au niveau politique }\end{array}$ \\
\hline Professionnel & $\begin{array}{l}\text { - Il agit selon la Convention pour les droits de l'enfant de l'ONU et le normes } \\
\text { actuelles d'éthique médicale }\end{array}$ \\
\hline Savant & $\begin{array}{l}\text { - Est prêt a apprendre tout au long de sa vie } \\
\text { - Comprend les données de surveillance épidémiologique locale et nationale } \\
\text { et évalue ces données de manière critique, afin de développer au niveau local } \\
\text { des offres adéquates pour la santé des enfants et adolescent-e-s. }\end{array}$ \\
\hline
\end{tabular}

Tableau 1. Rôle et compétences du/de la médecin scolaire, en tant que «expert-e en santé dans le cadre scolaire» (adapté d'après le «European Framework for quality standards in school health services and competences for school health professionals») $)^{5)}$

Un enfant avec des besoins particuliers mobilise généralement d'autres professionnel-le-s de la santé du milieu scolaire et les MS deviennent alors souvent les interlocutrices et interlocuteurs sur place. Une accessibilité à bas seuil rend possible l'accès à des bilans de santé aussi à des parents isolés, à des familles nouvellement arrivées ou à des mineur-e-s non accompagné-e-s - souvent abandonné-e-s à elles/eux-mêmes. Cette tâche est assumée par un service public, comme le service de santé scolaire, en collaboration avec les partenaires du réseau local. (Tableau 2)

Dans le cas de Diego:

Après une discussion avec l'administration scolaire, on convoque rapidement une réunion pour présenter la situation et mettre en place les premières mesures: un soutien en classe, une appréciation plus approfondie de ses difficultés avec les camarades de classe, ensuite un entretien de la MS et la direction de l'école avec la maman. Il est frappant que Diego n'arrive pas à se concentrer sur quelque chose, tant à la maison qu'à l'école; la maman fait des observations dans ce sens depuis longtemps, sans pouvoir les décrire. Cela nous amène à proposer un bilan psychologique afin de dépister d'éventuels problèmes développementaux et pouvoir ainsi suivre le développement et le comportement social de Diego en classe.

Cette situation met aussi en évidence le défi qui consiste à trouver un équilibre entre besoins individuels et exigences d'une collectivité, la classe d'école: il s'agit de favoriser et stimuler de manière ciblée le potentiel et l'épanouissement tant de l'individu que de l'ensemble.

Les MS ont, d'après A. Tschumper, «la tâche de soutenir, au niveau santé, le développement du potentiel d'apprentissage des enfants. Elles/ils rencontrent individuellement l'enfant et constatent son état de santé à l'école, que ce soit lors des examens de dépistage ou lors d'une consultation pour des pro- 


\section{Formation continue}

Champs d'activité de l'infirmier-e scolaire

et des services de santé scolaire

Examens de santé scolaire préventifs centré sur le développement, tests visuel et auditif, évaluation de la posture et du dos

Rappels de vaccins et campagnes de vaccination si nécessaire

Éducation à la santé en collaboration avec l'école
Qui est responsable? Comment est-ce organisé au niveau local?

Dispositions cantonales:

MS exerçant à titre principal ou accessoire Infirmier-e scolaire

Sur la base des recommandations nationales pour les vaccinations et des dispositions cantonales: MS exerçant à titre principal ou accessoire Infirmier-e scolaire

Selon les cantons et/ou la commune généralement délégué au service de santé scolaire, à des organisations de prévention ou à des médecins installés avec fonction de MS

Selon les dispositions cantonales ou communales (dépendent en pratique souvent des ressources de la commune), par l'infirmier-e scolaire ou le MS

Consultations avec l'infirmier-e scolaire, si nécessaire avec le MS

Niveau commune: service de santé scolaire, médecins installés avec fonction de MS, exige beaucoup d'initiative personnelle

Mise en place de réseaux avec les partenaires intra- et extrascolaires

Personne de contact pour les enseignant-e-s et/ou les parents d'enfants avec des besoins particuliers

Dispositions cantonales, interprétées au niveau communal: service de santé scolaire, MS exerçant à titre principal ou accessoire

Surveillance épidémiologique dans le cadre scolaire

Dispositions cantonales:

MS exerçant à titre principal ou accessoire Infirmier-e scolaire

Tableau 2. Répartition des tâches et compétences des services de santé scolaire, avec des différences régionales.

blèmes spécifiques (absentéisme, questions sur la santé dans le contexte scolaire, mise en danger du bien-être de l'enfant, etc.)». Et A. Tschumper d'ajouter: «Par la possibilité d'une approche d'enquêteur et universelle, le service de santé scolaire atteint aussi des familles et des enfants qui, pour différentes raisons, ont perdu ou n'ont pas encore établi de contact avec un-e médecin de famille»3).

L'activité des MS ne touche pas seulement à l'individu mais ils ont aussi une fonction de «médecin d'entreprise» dans le «lieu de vie et de travail école». Ainsi les MS et les infirmier-e-s scolaires contribuent à façonner un climat physique et psychique sain de l'école, p.ex. lors de l'aménagement de la cour d'école. Leur présence à l'école permet un accès à bas seuil pour tout le monde et d'atteindre, par leur approche «d'enquêtrice ou d'enquêteur», celles et ceux qui tombent entre les mailles du système ambulatoire.

Les services de santé scolaire conseillent écoles et autorités et «flairent» de nouvelles tendances

Les collaboratrices et collaborateurs des services de santé scolaire donnent des conseils concernant l'hygiène et les concepts de protection contre les ma- ladies infectieuses - redevenus d'actualité avec la pandémie Corona en 2020. Elles/ils peuvent aussi être consulté-e-s pour des questions de harcèlement ou de violence à l'école, psychosociales ou touchant au genre. Parfois leur collaboration est précieuse aussi concernant l'offre alimentaire et en activités physiques, ou pour l'élaboration de concepts ergonomiques des installations scolaires, les élèves passant le plus clair de leur journée dans les salles de classe.

Les MS sont des observatrices et observateurs de tendances dans le domaine de la santé, du préscolaire jusqu'au terme de la scolarité, et peuvent rapidement constater des problèmes dans le cadre scolaire et mettre en place - si nécessaire en collaboration avec les autorités locales - d'éventuelles solutions. Cette fonction correspond aux exigences pour l'âge scolaire formulées dans le Manifeste pour la santé des enfants et adolescents ${ }^{6}$. L'exemple suivant illustre cette tâche:

Depuis la réouverture des écoles après la première vague Corona entre mars et juin 2020, dans une école secondaire accueillant quotidiennement jusqu'à 800 élèves, a été constatée une augmentation d'angoisses jusqu'à des états d'anxiété chez certains élèves d'abord, puis des attaques de panique dans des classes entières. 
Les enseignant-e-s et les élèves ressentent une «électricité» insupportable se répandre. On fait appel au MS.

Plusieurs ateliers et interventions dans les classes, des entretiens individuels ainsi que des approches centrées sur le corps, pour les élèves et pour le corps enseignant, ont contribué à faire diminuer les angoisses, à baisser le niveau de stress; les élèves ont été pris en charge par l'infirmière scolaire ou le MS tout en restant intégrés dans leur cadre scolaire.

Par leur fonction responsable dans un service publique, les MS sont légitimé-es à rendre attentifs les directions et les autorités scolaires ou les responsables politiques à la nécessité d'agir. En collaboration avec la direction de l'école, elles/ils peuvent proposer les mesures nécessaires et assister dans un esprit interdisciplinaire à leur mise en œuvre. Le rôle des MS pour la santé des adolescent-e-s a été décrit en détail dans Paediatrica vol. 30/47).

Une autre tâche importante des MS est la lutte contre les maladies infectieuses et les vaccinations. L'information des adolescent-e-s autant que la discussion ouverte avec des parents sceptiques vis-à-vis des vaccins prennent une place prépondérante. Les campagnes de vaccination et les rappels de vaccins sont des démarches promues par le canton, ayant pour but, par un accès à bas seuil, une couverture vaccinale la plus large possible.

\section{Diversité des modèles}

L'activité des MS est ancrée dans la plupart des cantons dans la législation sur la santé et l'éducation. Les conditions cadres cantonales délèguent aux communes la responsabilité de doter et organiser le service de santé scolaire. Cela a pour conséquence des modèles très variés, analysés de manière critique dans un travail de master en 20178). On y constate des organisations et des offres très hétérogènes d'un canton à l'autre. Cela se montre p.ex. dans la réalisation et la teneur des examens de dépistage et des vaccinations, effectués à certains endroits par le service de médecine scolaire, alors qu'à d'autres ils sont délégués aux médecins installés. L'auteure met en évidence quatre modèles différents, allant du modèle public classique au modèle délégué au secteur privé, et mentionne comment les examens de santé et les vaccinations sont proposés dans le cadre scolaire dans les différents cantons et régions linguistiques. (Figure 3)

\section{Réseau national}

ScolarMed $\mathrm{CH}$, une association regroupant toutes et tous les professionnel-les actives et actifs dans les services de santé scolaire, propose un site web (scolarmed.ch), une plateforme d'échange pour ses membres ainsi que des journées scientifiques annuelles. Cette organisation, entretemps bien établie, contribue ainsi à assurer une prise en charge adéquate des élèves par la médecine scolaire en Suisse.

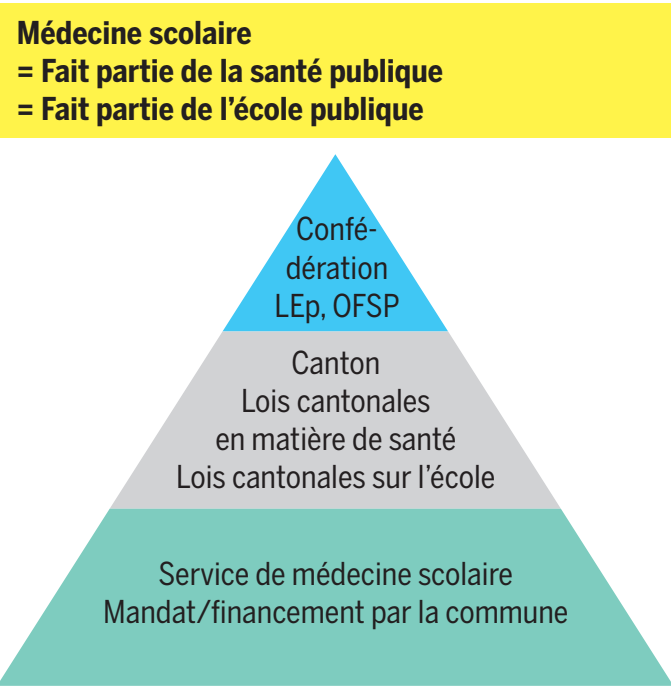

Figure 3. Services de santé scolaire: compétences aux différents niveaux (avec l'accord de la Dresse A. Tschumper).

Elle se compose de trois groupes professionnels, les médecins scolaires exerçant à titre principal ou accessoire, les infirmier-e-s scolaires (la plupart en Suisse romande) et les professionnel-le-s de la santé exerçant en privé (la plupart en Suisse alémanique). ScolarMed $\mathrm{CH}$ est ouverte aux membres individuels et au services cantonaux ${ }^{9}$ ) et est liée à pédiatrie suisse.

\section{Résumé et conclusion:} dans quelle mesure les services de santé médicale contribuent-ils actuellement à la santé des enfants et adolescents?

Les MS favorisent l'égalité des chances dans les écoles (enfantines):

- par la prise en charge médicale scolaire, tous les enfants et adolescent-e-s sont activement suivis (en respectant le libre choix du médecin) dans le cadre de l'école

- le «double regard», tant sur l'individu que sur la collectivité, contribue à la meilleure intégration possible aussi de celles et ceux qui apportent moins de «bagage» ou souffrent d'une maladie chronique, d'un handicap ou de troubles du développement, et qui doivent s'intégrer dans une génération multiculturelle et numérisée afin de bénéficier des mêmes chances professionnelles.

Par leur expertise et proximité, les MS peuvent exercer une influence positive sur le «lieu de travail et de vie école», dont le rôle est important pour une croissance et un développement sains:

- les MS se trouvent souvent au cœur de l'action, observent les tendances et suivent le développement 


\section{Formation continue}

des enfants et adolescent-e-s tout au long de la scolarité. Ils peuvent donc réagir rapidement à de nouvelles tendances, contribuer à la promotion de la santé et conseiller les directions des écoles et le corps enseignant sur des questions touchant à la santé.

- elles/ils complètent l'offre en médecine préventive des pédiatres et médecins de famille. La collaboration entre elles/eux garantit les meilleures chances d'un développement sain.

Dans ce sens les MS de nos jours poursuivent l'idéal des pionnières et pionniers de la médecine scolaire, en l'adaptant continuellement et de manière innovante aux défis actuels.

Cette tâche exige avant tout un travail interdisciplinaire, sans perdre de vue l'enfant, les adolescent-e-s, les élèves et leurs besoins.

Pour la bibliographie, veuillez consulter notre version en ligne de l'article.

Nous remercions la Drsse Annemarie Tschumper pour ses précieux commentaires et la mise à disposition des figures et graphiques.

\section{Auteures}

Dr med Tina Huber-Gieseke, médecine scolaire/service des écoles de la Ville de Fribourg Dr. med. Susanne Stronski Huwiler, Gesundheitsdienst Stadt Bern und Abteilung Neuropädiatrie, Entwicklung und Rehabilitation, Inselspital Bern

Les auteures n'ont déclaré aucun lien financier ou personnel en rapport avec cet article. 